\title{
Downregulation of TACC3 inhibits tumor growth and migration in osteosarcoma cells through regulation of the NF- $\mathrm{NB}$ signaling pathway
}

\author{
CONGRAN ZHAO, XIAOFENG HE, HENG LI, JIHUI ZHOU, XIUYING HAN, \\ DONGJUN WANG, GUOFENG TIAN and FUGE SUI
}

Department of Orthopedics, Daqing Longnan Hospital, Daqing, Heilongjiang 163453, P.R. China

Received November 14, 2016; Accepted December 21, 2017

DOI: $10.3892 / \mathrm{ol} .2018 .8262$

\begin{abstract}
TACC3, a member of the transforming acidic coiled-coil protein (TACC) family, is a multifunctional protein that is involved in various biological functions, including proliferation and differentiation of tumor cells, cancer progression and metastasis. The aims of the present study were to examine whether TACC 3 expression is associated with the proliferation and migration of osteosarcoma (OS) cells and to investigate the potential underlying molecular mechanisms of TACC 3 in OS. First, the levels of mRNA and protein expression in OS cell lines by reverse transcription-quantitative polymerase chain reaction and western blotting, respectively were examined. Second, the effects of TACC3 knockdown and overexpression on the proliferative, migratory and invasive capacities of OS cells were investigated. Finally, western blot analysis was employed to detect the potential mechanism of TACC 3 in osteosarcoma. TACC 3 expression was significantly increased in osteosarcoma tissues and cell lines, compared to matched controls. The knockdown of TACC 3 was able to significantly inhibit the proliferation, migration and invasion of osteosarcoma cells, whereas the overexpression of TACC3 was able to promote cell proliferation and migration. Mechanistically, TACC3 may promote the migration and invasion of osteosarcoma cells via through nuclear factor- $\kappa \mathrm{B}$ signaling. These data suggest that TACC3 has an important part in the progression of osteosarcoma and may serve as a potential target for gene therapy.
\end{abstract}

Correspondence to: Dr Fuge Sui, Department of Orthopedics, Daqing Longnan Hospital, 35 Aiguo Road, Daqing, Heilongjiang 163453, P.R. China

E-mail: fuge.sui@hotmail.com

Abbreviations: TACC, transforming acidic coiled-coil protein; OS, osteosarcoma; OS, overall survival

Key words: transforming acidic coiled-coil protein, osteosarcoma, migration, proliferation

\section{Introduction}

Osteosarcoma (OS) is one of the most common malignant bone tumors, which occurs frequently in children and adolescents (1). Furthermore, OS has a strong tendency to metastasize (1). Despite intensive efforts to identify novel treatment strategies, survival rates have not improved in the past two decades (2). There has been considerable research on the molecular mechanism underlying OS, and a growing body of evidence has revealed that the regulation of oncogenes and tumor suppressor genes is vital in the development and progression of OS (3-5). Therefore, there is important clinical value in identifying the molecular targets and agents to improve the diagnosis and prognosis of OS.

The tumor protein D52 (TACC3) gene was first identified $\sim 20$ years ago (6). TACC3, located at the chromosome 4p16.3, and stabilizes and organizes the mitotic spindle to allow for proper chromosomal segregation (7). There are three TACC proteins that are identified in humans: TACC1, TACC 2 and TACC3. TACC3, originally isolated from the 4 p16.3 region, is an Aurora and integrin-linked kinase target, strongly concentrated at centrosomes throughout the cell cycle and identified as a member of the centrosomal protein family that can regulate the formation of microtubules (8). Multiple studies have revealed that TACC3 is a multifunctional protein involved in various biological functions, including cell survival, proliferation, migration, invasion, DNA repair, exocytosis and vesicle trafficking $(9,10)$. Previous studies have reported that TACC3 is overexpressed in several types of cancer, including, esophageal squamous cell carcinoma, hepatocellular carcinoma, lung, pancreatic, cervical and gastric cancer $(11,12)$. However, to the best of our knowledge, this is the first study to assess the expression of TACC 3 in osteosarcoma and to investigate the molecular mechanism underlying the TACC3-mediated regulation of tumor progression.

The nuclear factor $-\kappa \mathrm{B}(\mathrm{NF}-\kappa \mathrm{B})$ signaling pathway is involved in immune and inflammatory responses, including, tumorigenic processes. The deregulated activation of NF- $\kappa \mathrm{B}$ is associated with cancer $(13,14)$. Previous research studies have reported that $\mathrm{NF}-\kappa \mathrm{B}$ affects a number of tumor malignant behaviors, including proliferation, invasion and metastasis by regulating the expression of several genes relevant to 
tumorigenesis. For example, $\mathrm{NF}-\kappa \mathrm{B}$ upregulates the expression of genes (cyclin D1 and c-Myc), which are involved in anti-apoptotic processes and the regulation of cell cycle $(15,16)$. In osteosarcoma, previous research has confirmed that the excessive activation of the $\mathrm{NF}-\kappa \mathrm{B}$ signaling pathway promotes the proliferation and metastasis of tumor cells and increases resistance to chemotherapy (17). Tang et al (18) also reported an original mechanism for the involvement of the $N F-\kappa B$ signaling pathway in glycogen synthase kinase-3 $\beta$-mediated regulation of cell survival in osteosarcoma. Therefore, it was proposed that the $\mathrm{NF}-\kappa \mathrm{B}$ pathway may be involved in the proliferation and metastasis process of cancer.

The purpose of the present study was to investigate the regulatory role of TACC 3 in the proliferation, migration and invasion of osteosarcoma cells, including, the potential molecular mechanism by which TACC3 exerts its effects.

\section{Materials and methods}

Cell culture. The human osteosarcoma cell lines U2-OS, MG63 and normal human osteoblasts (NHOst) were obtained from the American Type Culture Collection (ATCC; Manassas, VA, USA). The 143B and SAOS cells were obtained from the Type Culture Collection of the Chinese Academy of Sciences (Shanghai, China). All cells were cultured in RPMI-1640 (Gibco; Thermo Fisher Scientific, Inc., Waltham, MA, USA) medium supplemented with $10 \%$ fetal bovine serum (FBS; Gibco, Thermo Fisher Scientific, Inc.) in a humidified incubator with $5 \% \mathrm{CO}_{2}$ at $37^{\circ} \mathrm{C}$.

Patient information and tissue specimens. The present study was approved by the Institutional Ethics Committee and Review Board of the Daqing Longnan Hospital (Daqing, China). All study participants or their legal guardian, provided written informed consent prior to enrollment. A total of 36 osteosarcoma specimens and matched adjacent noncancerous osteosarcoma tissues were obtained who had undergone resection for osteosarcoma between 2013 and 2015. Their median age was 18 years (range, 13-38 years) and the male:female ratio was 23:13. Following resection, matched fresh tissues were immersed immediately in RNAlater ${ }^{\circledR}$ (Ambion, Thermo Fisher Scientific, Inc.), kept overnight at $4^{\circ} \mathrm{C}$ and then stored at $-80^{\circ} \mathrm{C}$ until RNA isolation.

Reverse transcription-quantitative polymerase chain reaction $(R T-q P C R)$. Total RNA from cells and tissues were extracted using the TRIzol solution (Invitrogen; Thermo Fisher Scientific, Inc.) according to manufacturer's instructions. RNA concentration and purity were determined by absorbance at $260 \mathrm{~nm}$ using a NanoDrop ND-1000 spectrophotometer (NanoDrop Technologies; Thermo Fisher Scientific, Inc., Wilmington, DE, USA). RT was performed on $2 \mu \mathrm{g}$ total RNA sample using M-MLV reverse transcriptase kit (Promega Corporation, Madison, WI, USA) at $37^{\circ} \mathrm{C}$ for $60 \mathrm{~min}$ according to the manufacturer's protocol. Newly synthesized cDNA was amplified by RT-qPCR to enable the expression levels of TACC 3 to be detected. The primers used were as follows: TACC3 forward, 5'-CCTCTTCAAGCGTTT TGAGAAAC-3' and reverse, 5'-GCCCTCCTGGGTGATCCT T-3'; GAPDH, forward, 5'-CTCCTCCTGTTCGACAGTCAG
C-3', and reverse, 5'-CCCAATACGACCAAATCCGTT-3'. qPCR amplification was performed in an ABI 7900HT Real-time PCR system (Thermo Fisher Scientific, Inc.). The PCR was conducted in a final volume of $15 \mu \mathrm{l}$, consisting of $7.5 \mu \mathrm{l}$ of $2 \mathrm{X}$ SYBR Green Master Mix (Invitrogen; Thermo Fisher Scientific, Inc.), $2 \mu \mathrm{l}$ of each primer (1.5 pmol/ $/ \mu \mathrm{l}), 0.5 \mu \mathrm{l}$ sample cDNA and $5 \mu \mathrm{l}$ water. The thermocycling conditions were as follows: One cycle of $95^{\circ} \mathrm{C}$ for $10 \mathrm{~min}$, followed by $95^{\circ} \mathrm{C}$ for $30 \mathrm{sec}$ and $60^{\circ} \mathrm{C}$ for $60 \mathrm{sec}$ for 45 cycles. The relative expression levels of TACC 3 were normalized to that of the internal control gene, GAPDH. The data were analyzed using the comparative threshold cycle $\left(2^{-\Delta \Delta \mathrm{Cq}}\right)$ method (19).

Western blot analysis. Osteosarcoma cell lines were lysed in RIPA lysis buffer (Beyotime Institute of Biotechnology, Shanghai, China). The lysates were harvested by centrifugation $\left(12,000 \mathrm{x}\right.$ g for $20 \mathrm{~min}$ at $4^{\circ} \mathrm{C}$. The protein samples $(20 \mu \mathrm{g})$ were resolved in $12 \%$ sodium dodecyl sulfate polyacrylamide gel by electrophoresis and transferred to a polyvinylidene difluoride (PVDF) membrane. After blocking non-specific binding sites for 60 min with $8 \%$ non-fat milk in TBST, the membranes were incubated with primary antibodies, rabbit polyclonal anti-TACC3 (1:1,000; Abcam, Cambridge, UK; cat. no. ab134154), anti-p65 (1:1,000; Cell Signaling Technology, Inc., Danvers, MA, USA; cat. no. 8242) anti-matrix metalloproteinase-9 (MMP9; 1:1,000; Cell Signaling Technology, Inc.; cat. no. 13667), anti-cellular FLICE-like inhibitory protein (c-FLIP; 1:500; Cell Signaling Technology, Inc.; cat. no. 56343) or GAPDH (1:10,000; ProteinTech Group, Inc., Chicago, IL, USA; cat. no. 10494-1-AP) overnight at $4^{\circ} \mathrm{C}$. The membranes were washed four times with TRIS-buffered saline with Tween-20 for 10 min. After washing, the membranes were probed with horseradish peroxidase-conjugated goat anti-rabbit antibody (1:5,000; EMD/Merck KGaA, Darmstadt, Germany; cat. no. AP307P) at room temperature for $1 \mathrm{~h}$, and an enhanced chemiluminescence detection kit (Cell Signaling Technology, Inc.), was used to visualize the proteins. Band intensity was analyzed using the Quantity One software 4.6.2 (Bio-Rad Laboratories, Inc., Hercules, CA, USA).

Transfection of osteosarcoma cells. For TACC3 functional analysis, MG63 and U2-OS cells were transfected with TACC3 siRNA or pcDNA3.1 TACC3 plasmid using Lipofectamine ${ }^{\circledR}$ iMAX reagent (Invitrogen; Thermo Fisher Scientific, Inc.), according to the manufacturer's instructions. MG63 and U2-OS $\left(2 \times 10^{5}\right)$ cells were seeded into 6-well plates, incubated for $24 \mathrm{~h}$ and then transfected with $12.5 \mathrm{nM}$ RNA duplex and $5 \mu 1$ Lipofectamine RNAiMAX (Invitrogen; Thermo Fisher Scientific, Inc.) according to the manufacturer's instructions. The cells were harvested for further experiments after 48 or 72 h. siRNA oligo-ribonucleotides were purchased from Guangzhou RiboBio Co., Ltd., (Guangzhou, China). The effective siRNA sequences were as follows: TACC3-siRNA-(5'-GCATGCACGGTGCAAATGA-3'). In addition, the cDNA of the human TACC 3 gene, a fragment encoding the TACC3-sequence plus 1,439 bp at both 5'- and 3'-flanking regions was amplified with the primers: 5'-GAG GATCCCCGGGTACCGGTCGCCACCATGAGTCTGCAG GTCTTAAACGAC-3' (forward) and 5'-TCCTTGTAGTCC ATACCGATCTTCTCCATCTTGGAGATGAG-3' (reverse) 
A

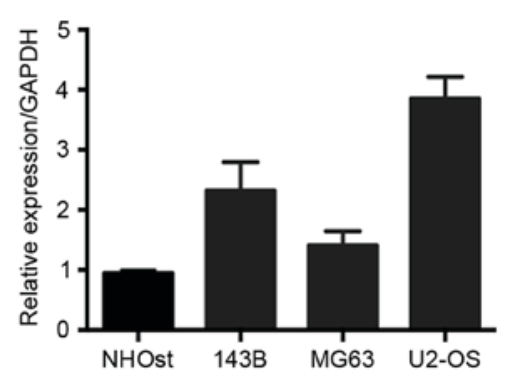

B

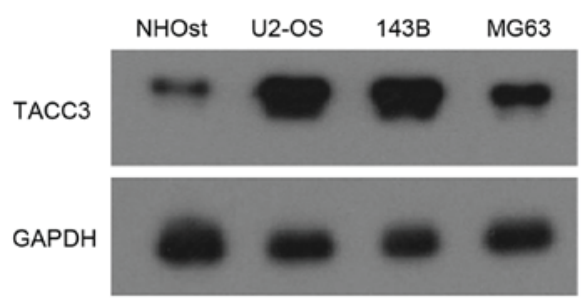

C

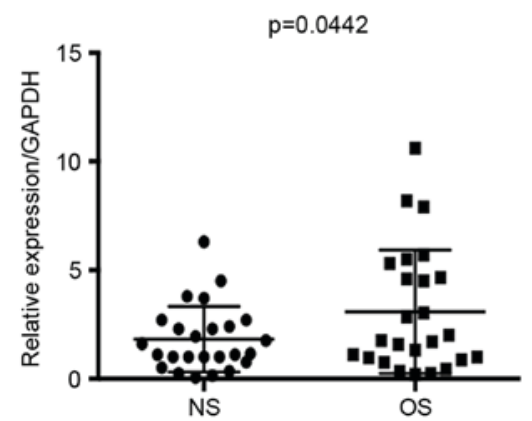

Figure 1. (A) mRNA level of TACC3 was examined in osteosarcoma cell lines using RT-qPCR. (B) Protein levels of TACC3 were determined by western blot analysis in various osteosarcoma cell lines. (C) The mRNA level of TACC 3 in osteosarcoma tissues and normal matched non-cancerous tissue as assessed by RT-qPCR. NS, non-cancerous tissue; OS, osteosarcoma; TACC3, transforming acidic coiled-coil protein; RT-qPCR, reverse transcription-quantitative polymerase chain reaction.

by PCR from human genomic DNA and then cloned into the AgeI/NheI sites of GV358.

Cell proliferation assay. MTS assay was used to analyze the proliferation of TACC3 siRNA-transfected and TACC3 lentivirus-transfected MG63 and U2-OS cells. The cells were cultured in 96-well plates at 2,500 cells/well. At each time-point $(0,24,48,72,96$ and $120 \mathrm{~h})$, the cells incubated with $20 \mu \mathrm{l}$ MTS (5 mg/ml; Sigma-Aldrich, Merck KGaA) for $4 \mathrm{~h}$ in $5 \% \mathrm{CO}_{2}$ at $37^{\circ} \mathrm{C}$. Finally, the A490 value of each sample was determined using a microplate reader. Statistical analyses were carried out using a two-tailed unpaired Student's t-test.

Cell migration and invasion assays. Cell migration and invasion assays were performed using polycarbonate filters (pore size, 8-mm) in 24-well Transwell chambers (Corning Inc., Corning, NY, USA). The cells were seeded at $5 \times 10^{4}$ in $200 \mu \mathrm{l}$ serum-free medium in the upper chamber, and $500 \mu \mathrm{l}$ RPMI 1640 medium containing 5\% FBS was placed in the lower chamber. Following incubation for $24 \mathrm{~h}$ at $37^{\circ} \mathrm{C}$, the cells remaining in the upper chamber were removed with cotton
A

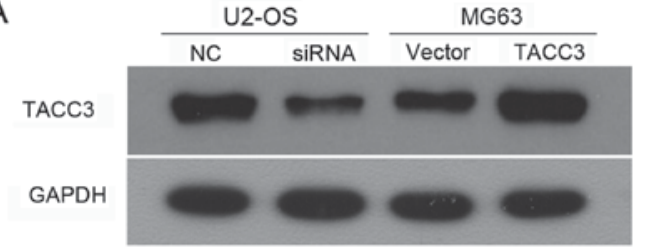

B
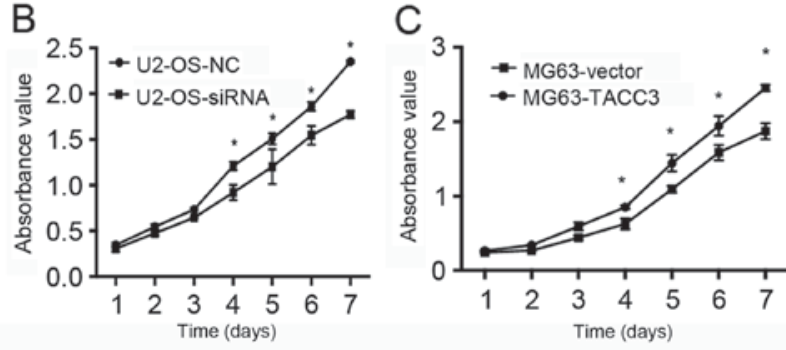

Figure 2. (A) Protein levels of TACC3 were determined using western blot analysis in osteosarcoma MG63 cells that were transfected with TACC3 plasmid and in U2-OS cells that were transfected with TACC3 siRNA. (B) MTS assay was used to determine the proliferation of TACC3 siRNA-transfected U2-OS cells. ${ }^{*} \mathrm{P}<0.05$ vs. the control; (C) MTS assay was used to determine the proliferation of TACC3 plasmid-transfected MG63 cells. ${ }^{*} \mathrm{P}<0.05$ vs. control; NC, negative control; siRNA, small-interfering RNA; TACC3, transforming acidic coiled-coil protein.

swabs. The cells that migrated to the lower surface of the membrane were fixed with methanol and stained with crystal violet at room temperature for $15 \mathrm{~min}$. The cells in $\geq 5$ random fields of view at x100 magnification were counted (CKX41; Olympus Corporation, Tokyo, Japan). Each experiment was performed in triplicate. Statistical analyses were performed using the two-tailed unpaired Student's t-test.

Statistical analyses. Statistical analyses were conducted using SPSS (version 18.0; SPSS, Inc., Chicago, IL, USA). The comparisons between groups were analyzed using two-tailed unpaired Student t-test, unless otherwise specified. The data are expressed as the mean \pm standard deviation. $\mathrm{P}<0.05$ was considered to indicate a statistically significant difference.

\section{Results}

TACC3 is upregulated in osteosarcoma tissues. In the present study, the levels of TACC3 in osteosarcoma tissues and osteosarcoma cell lines U2-OS, MG63, 143B and SAOS, were examined. RT-qPCR and western blot analysis indicated that TACC 3 mRNA and protein expression levels were markedly higher in the osteosarcoma cell lines compared with normal NHOst cell line, particularly in U2-OS cells (Fig. 1A and B). In addition, the mRNA expression level of TACC3 was upregulated in osteosarcoma tissues compared with the normal adjacent tissues (Fig. 1C). The present data suggested that increased TACC 3 expression is associated with osteosarcoma.

TACC3 promotes the proliferation of osteosarcoma cells in vitro. In order to examine the function of TACC 3 in the progression of osteosarcoma in vitro, U2-OS cells were transfected with TACC3 siRNA, and MG63 cells were infected with TACC3 lentivirus to silence and overexpress TACC3, respectively. Western blot analysis was used to 
A

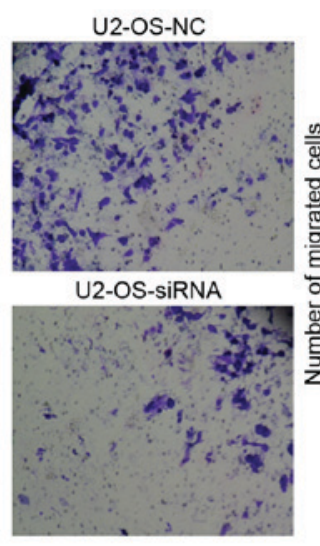

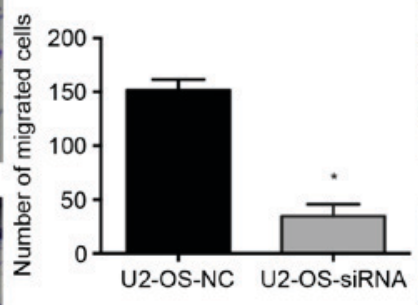

C

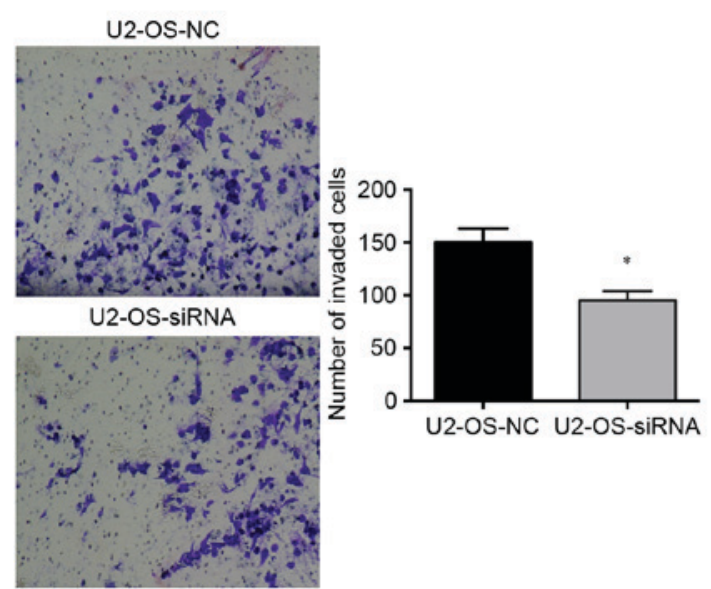

B

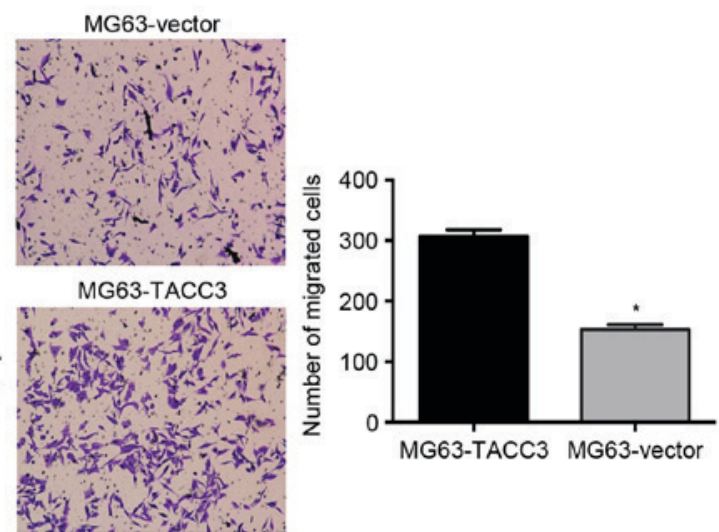

D

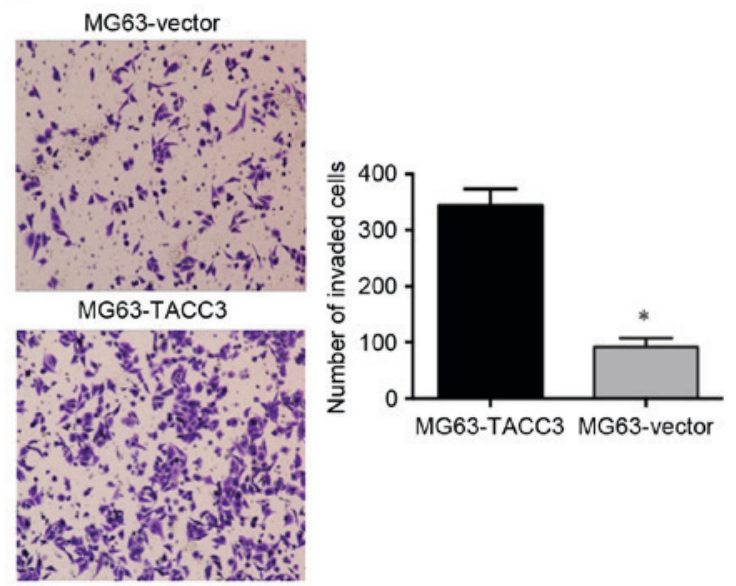

Figure 3. Transwell assay was performed to determine migration and invasion in osteosarcoma MG63 cells that were transfected with TACC3 plasmid and U2-OS cells that were transfected with TACC 3 siRNA. (A) Migration assay of TACC3 siRNA-transfected U2-OS cells. "P<0.05 vs. the control. (B) Migration assay of TACC 3 plasmid-transfected MG63 cells. " $\mathrm{P}<0.05$ vs. the control. (C) Invasion assay of TACC 3 siRNA-transfected U2-OS cells. ${ }^{*} \mathrm{P}<0.05$ vs. the control. (D) Invasion assay of TACC3 plasmid-transfected MG63 cells. Images are shown on the left (x100 magnification). "P<0.05 vs. the control.

verify knockdown and overexpression efficiency in U2-OS cells and MG63 cells. The results indicated that the expression levels of TACC3 were upregulated in MG63 cells that were transfected with TACC 3 lentivirus, and reduced in U2-OS cells that were transfected with TACC3 siRNA compared with the control cells (Fig. 2A). To examine the effect of TACC 3 expression on tumorigenicity, the growth of MG63 cells that were infected with TACC3 lentivirus or U2-OS cells transfected with TACC3 siRNA was assessed. The results demonstrated a significant decrease in the growth rate of TACC3 siRNA-transfected cells compared with the control cells $(\mathrm{P}<0.05$; Fig. 2B). The overexpression of TACC 3 in MG63 cell line significantly promoted the cell proliferation rate compared with the control $(\mathrm{P}<0.05$; Fig. $2 \mathrm{C})$. Therefore, these findings indicated that TACC 3 may serve a carcinogenic role in progression of osteosarcoma.

TACC 3 promotes the migration and invasion of osteosarcoma cells in vitro. To determine the role of TACC 3 in cell migration and invasion, Transwell assays of MG63 and U2-OS cells were performed in vitro. The silencing of TACC 3 expression significantly reduced the migration of U2-OS cells compared with the control cells $(\mathrm{P}<0.05$; Fig. 3A). Compared with the control

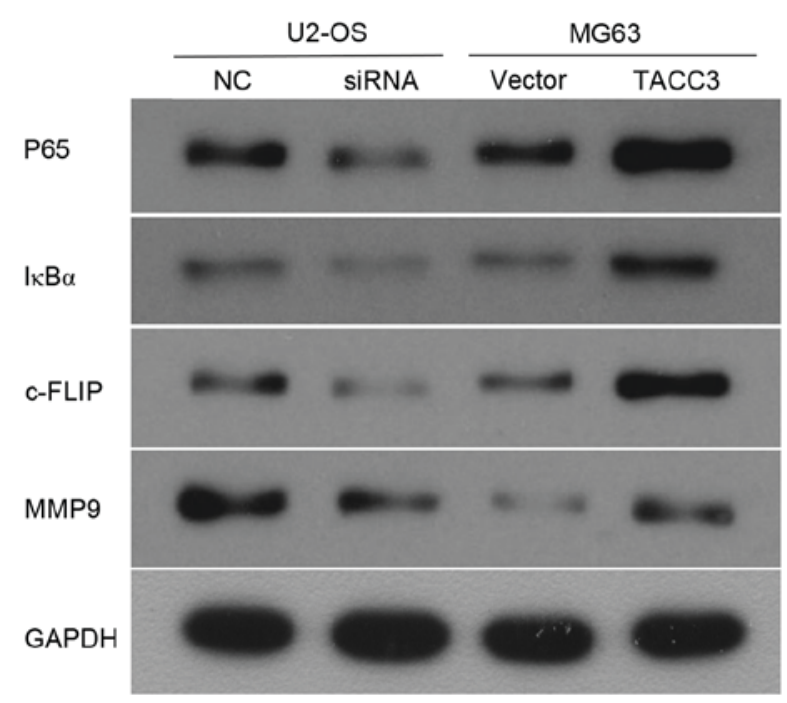

Figure 4. Western blot analysis to determine the protein expression of nuclear factor- $\mathrm{\kappa B}$ signaling pathway in osteosarcoma MG63 cells that were transfected with TACC 3 plasmid and in U2-OS cells that were transfected with TACC3 siRNA. GAPDH, glyceraldehyde 3-phosphate dehydrogenase; TACC3, transforming acidic coiled-coil protein 3; c-FLIP,

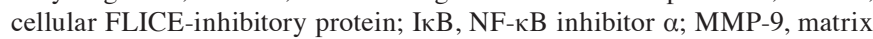
metalloproteinase-9; NC, negative control; siRNA, small-interfering RNA. 
cells, the overexpression of TACC 3 induced an increase in the migration of MG63 osteosarcoma cell line compared with control cells $(\mathrm{P}<0.05$; Fig. $3 \mathrm{~B})$. Consistent with the results of the migration assay, the invasion assay demonstrated that the knockdown of TACC3, significantly inhibited cell invasion $(\mathrm{P}<0.05$; Fig. 3C). The cell invasion assay also indicated that cell invasion was significantly increased when TACC3 was overexpressed $(\mathrm{P}<0.05$; Fig. 3D).

TACC 3 regulates the $N F-\kappa B$ signaling pathway in osteosarcoma cells. It has been reported that the activation of $\mathrm{NF}-\kappa \mathrm{B}$ promotes the development of osteosarcoma (18), which suggests that the effect of TACC 3 on osteosarcoma may be involved with the $\mathrm{NF}-\kappa \mathrm{B}$ signaling pathway. Therefore, a further aim of the present study was to investigate whether the upregulation of TACC 3 promotes metastasis in osteosarcoma through activation of the NF- $\mathrm{B}$ pathway. The western blot analysis revealed that the overexpression of TACC3 in MG63 cells increased the protein expression levels of IкB $\alpha$ and p65 (Fig. 4). Consequently, the target genes of NF- $\kappa$ B, including, MMP9 and c-FLIP were increased following TACC 3 overexpression. By contrast, the opposing expression patterns of these aforementioned genes were observed in TACC3-knocked down cells (Fig. 4).

\section{Discussion}

Previous studies have demonstrated that the transforming acidic coiled-coil protein (TACC) family members, notably TACC3, serve a critical function in tumor development and progression (20). However, the number of studies on the role of TACC3 in osteosarcoma is limited.

Therefore, there is an urgent requirement to identify novel molecular targets with therapeutic potential for the treatment of osteosarcoma. In the present study, it was confirmed that TACC3 is upregulated in osteosarcoma tissues and cell lines compared with normal NHOst cell line and adjacent noncancerous osteosarcoma tissues. The results from the present in vitro studies revealed that TACC3 promoted the proliferation, migration and invasion of osteosarcoma cells by activating the $\mathrm{NF}-\kappa \mathrm{B}$ signaling pathway.

Different oncogene expression profiles in osteosarcoma have been identified previously. TACC 3 have involved in the tumorigenesis of different cancer types $(21,22)$. The overexpression of TACC 3 has been verified to be associated with a poor overall survival (OS) and poor recurrence-free survival of patients with non-small cell lung cancer (23). Nahm et al (24) demonstrated that high protein levels of TACC3 were associated with histological differentiation, tumor size, microvascular invasion and pathological tumor-node metastasis stage in 188 hepatocellular carcinoma (HCC) tissue specimens. In addition, the authors demonstrated that the silencing of TACC3 by siRNA decreased the invasive ability of HCC cells, indicating that TACC3 may be a major contributory factor in tumor development.

TACC3 is involved with several types of human cancer, including osteosarcoma. However, the exact role of TACC3 in the regulation of proliferation and migration of osteosarcoma cells has not been clarified. In the present study, it was revealed that the knockdown of TACC3 significantly suppressed the proliferation and migration of osteosarcoma cells, and the overexpression of TACC3 notably enhanced the proliferation and migration of osteosarcoma cells. Consequently, the results from the present study suggest that TACC3 may serve an oncogenic role in the regulation of osteosarcoma cells.

It has previously been reported that TACC3 is able to promote cell proliferation by regulating several signaling pathways. For example, Zhou et al (12) reported that TACC3 expression is frequently increased in HCC tumor tissues compared with matched non-cancerous samples. Furthermore, the knockdown of TACC3 suppressed tumor stem cell-like characteristics through the Wnt/ $\beta$-catenin and phosphatidylinositide 3-kinase/protein kinase B (PI3K/AKT) signaling pathways. Huang et al (25) reported that TACC3 is upregulated in human esophageal squamous cell carcinoma (ESCC) and promotes the proliferation, colony formation and migration of esophageal squamous cell carcinoma cells.

TACC3 may also act as a potential oncogene that promotes cell proliferation by epidermal growth factor (EGF)-mediated epithelial-mesenchymal transition (EMT) in cervical cancer cells (26). In accordance with these previous results, it was verified in the present study that TACC3 regulates the proliferation, migration and invasion of osteosarcoma cells. Therefore, targeting TACC3 may be an attractive strategy for the treatment of osteosarcoma. However, the specific regulatory mechanism of how TACC3 exerts its function remains unclear.

There were several limitations to the present study. Firstly, the scope of the experiments was limited to proliferation, migration and invasion. Therefore, future aims are to investigate the role of TACC3 with respect to the cell cycle, apoptosis and colony formation of osteosarcoma cell lines. Secondly, while it was demonstrated that TACC3 is able to modulate the proliferation and migration of osteosarcoma cells via the $\mathrm{NF}-\kappa \mathrm{B}$ signaling pathway, the effect of a $\mathrm{NF}-\kappa \mathrm{B}$ pathway inhibitor on TACC3 was not assessed. Finally, the present study focused solely on the association between TACC 3 and prognosis, and potential application in clinical practice were not investigated.

In conclusion, this present study demonstrates that TACC3 may act as a tumor oncogene in osteosarcoma. TACC 3 is able to promote the proliferation, migration and invasion of osteosarcoma cells via the $\mathrm{NF}-\kappa \mathrm{B}$ signaling pathway. Therefore, TACC 3 may be a potential therapeutic target for the treatment of osteosarcoma in the future.

\section{Competing interests}

The authors declare that they have no competing interests.

\section{References}

1. Mirabello L, Troisi RJ and Savage SA: Osteosarcoma incidence and survival rates from 1973 to 2004: Data from the Surveillance, Epidemiology, and End Results Program. Cancer 115: 1531-1543, 2009.

2. Ferrari S, Smeland S, Mercuri M, Bertoni F, Longhi A, Ruggieri P, Alvegard TA, Picci P, Capanna R, Bernini G, et al: Neoadjuvant chemotherapy with high-dose Ifosfamide, high-dose methotrexate, cisplatin, and doxorubicin for patients with localized osteosarcoma of the extremity: A joint study by the Italian and Scandinavian Sarcoma Groups. J Clin Oncol 23: 8845-8852, 2005. 
3. Lu J, Song G, Tang Q, Zou C, Han F, Zhao Z, Yong B, Yin J, $\mathrm{Xu} \mathrm{H}, \mathrm{Xie} \mathrm{X}$, et al: IRX1 hypomethylation promotes osteosarcoma metastasis via induction of $\mathrm{CXCL} 14 / \mathrm{NF}-\kappa \mathrm{B}$ signaling. J Clin Invest 125: 1839-1856, 2015.

4. Hou CH, Lin FL, Hou SM and Liu JF: Cyr61 promotes epithelial-mesenchymal transition and tumor metastasis of osteosarcoma by Raf-1/MEK/ERK/Elk-1/TWIST-1 signaling pathway. Mol Cancer 13: 236, 2014

5. Tsai HC, Su HL, Huang CY, Fong YC, Hsu CJ and Tang CH: CTGF increases matrix metalloproteinases expression and subsequently promotes tumor metastasis in human osteosarcoma through down-regulating miR-519d. Oncotarget 5: 3800-3812, 2014.

6. Still IH, Vince P and Cowell JK: The third member of the transforming acidic coiled coil-containing gene family, TACC3, maps in $4 \mathrm{p} 16$, close to translocation breakpoints in multiple myeloma, and is upregulated in various cancer cell lines. Genomics 58: 165-170, 1999 .

7. Kiemeney LA, Sulem P, Besenbacher S, Vermeulen SH, Sigurdsson A, Thorleifsson G, Gudbjartsson DF, Stacey SN Gudmundsson J, Zanon C, et al: A sequence variant at 4p16.3 confers susceptibility to urinary bladder cancer. Nat Genet 42 : 415-419, 2010

8. Hood FE and Royle SJ: Pulling it together: The mitotic function of TACC3. Bioarchitecture 1: 105-109, 2011.

9. Sadek CM, Pelto-Huikko M, Tujague M, Steffensen KR, Wennerholm M and Gustafsson JA: TACC3 expression is tightly regulated during early differentiation. Gene Expr Patterns 3: 203-211, 2003

10. Yao R, Natsume Y and Noda T: TACC3 is required for the proper mitosis of sclerotome mesenchymal cells during formation of the axial skeleton. Cancer Sci 98: 555-562, 2007.

11. Capelletti M, Dodge ME, Ercan D, Hammerman PS, Park SI, Kim J, Sasaki H, Jablons DM, Lipson D, Young L, et al: Identification of recurrent FGFR3-TACC3 fusion oncogenes from lung adenocarcinoma. Clin Cancer Res 20: 6551-6558, 2014.

12. Zhou DS, Wang HB, Zhou ZG, Zhang YJ, Zhong Q, Xu L, Huang YH, Yeung SC, Chen MS and Zeng MS: TACC3 promotes stemness and is a potential therapeutic target in hepatocellular carcinoma. Oncotarget 6: 24163-24177, 2015.

13. Zhao Z, Wu MS, Zou C, Tang Q, Lu J, Liu D, Wu Y, Yin J, Xie X, Shen J, et al: Downregulation of MCT1 inhibits tumor growth, metastasis and enhances chemotherapeutic efficacy in osteosarcoma through regulation of the NF- $\mathrm{KB}$ pathway. Cancer Lett 342 150-158, 2014.

14. Schon S, Flierman I, Ofner A, Stahringer A, Holdt LM, Kolligs FT and Herbst A: $\beta$-catenin regulates NF- $\kappa$ B activity via TNFRSF19 in colorectal cancer cells. Int J Cancer 135: 1800-1811, 2014

15. Bera A, Ghosh-Choudhury N, Dey N, Das F, Kasinath BS, Abboud HE and Choudhury GG: NFKB-mediated cyclin D1 expression by microRNA-21 influences renal cancer cell proliferation. Cell Signal 25: 2575-2586, 2013.
16. Shao N, Lu Z, Zhang Y, Wang M, Li W, Hu Z, Wang S and Lin Y: Interleukin-8 upregulates integrin 3 expression and promotes estrogen receptor-negative breast cancer cell invasion by activating the PI3K/Akt/NF-кB pathway. Cancer Lett 364: 165-172, 2015.

17. Korber MI, Staribacher A, Ratzenbock I, Steger G and Mader RM: NFкB-Associated pathways in progression of chemoresistance to 5-fluorouracil in an in vitro model of colonic carcinoma. Anticancer Res 36: 1631-1639, 2016.

18. Tang QL, Xie XB, Wang J, Chen Q, Han AJ, Zou CY, Yin JQ, Liu DW, Liang Y, Zhao ZQ, et al: Glycogen synthase kinase-3, $\mathrm{NF}-\kappa \mathrm{B}$ signaling, and tumorigenesis of human osteosarcoma. J Natl Cancer Inst 104: 749-763, 2012.

19. Livak KJ and Schmittgen TD: Analysis of relative gene expression data using real-time quantitative PCR and the 2(-Delta Delta C(T)) method. Methods 25: 402-408, 2001.

20. Ha GH, Park JS and Breuer EK: TACC3 promotes epithelial-mesenchymal transition (EMT) through the activation of PI3K/Akt and ERK signaling pathways. Cancer Lett 332: 63-73, 2013.

21. Lauffart B, Vaughan MM,Eddy R, Chervinsky D, DiCioccio RA, Black JD and Still IH: Aberrations of TACC1 and TACC3 are associated with ovarian cancer. BMC Women's Health 5: 8, 2005.

22. Yun M, Rong J, Lin ZR, He YL, Zhang JX, Peng ZW, Tang LQ, Zeng MS, Zhong Q and Ye S: High expression of transforming acidic coiled coil-containing protein 3 strongly correlates with aggressive characteristics and poor prognosis of gastric cancer. Oncol Rep 34: 1397-1405, 2015.

23. Jiang F, Kuang B, Que Y, Lin Z, Yuan L, Xiao W, Peng R, Zhang $X$ and $Z$ hang $X$ : The clinical significance of transforming acidic coiled-coil protein 3 expression in non-small cell lung cancer. Oncol Rep 35: 436-446, 2016.

24. Nahm JH, Kim H, Lee H, Cho JY, Choi YR, Yoon YS, Han HS and Park YN: Transforming acidic coiled-coil-containing protein 3 (TACC3) overexpression in hepatocellular carcinomas is associated with 'stemness' and epithelial-mesenchymal transition-related marker expression and a poor prognosis. Tumour Biol 37: 393-403, 2016.

25. Huang ZL, Lin ZR, Xiao YR, Cao X, Zhu LC, Zeng MS, Zhong Q and Wen ZS: High expression of TACC3 in esophageal squamous cell carcinoma correlates with poor prognosis. Oncotarget 6: 6850-6861, 2015.

26. Ha GH, Kim JL and Breuer EK: TACC3 is essential for EGF-mediated EMT in cervical cancer. PLoS One 8: e70353, 2013.

(i) $\Theta$ This work is licensed under a Creative Commons Attribution-NonCommercial-NoDerivatives 4.0 International (CC BY-NC-ND 4.0) License. 